Swarthmore College

Works

$4-10-2000$

\title{
A Single Circumbinary Disk In The Hd 98800 Quadruple System
}

D. W. Koerner

Eric L.N. Jensen

Swarthmore College, ejensen1@swarthmore.edu

K. L. Cruz

T. B. Guild

K. Gultekin

Follow this and additional works at: https://works.swarthmore.edu/fac-physics

Part of the Astrophysics and Astronomy Commons

Let us know how access to these works benefits you

\section{Recommended Citation}

D. W. Koerner, Eric L.N. Jensen, K. L. Cruz, T. B. Guild, and K. Gultekin. (2000). "A Single Circumbinary Disk In The Hd 98800 Quadruple System". Astrophysical Journal. Volume 533, Issue 1. L37-L40. DOI: 10.1086/ 312593

https://works.swarthmore.edu/fac-physics/8

This work is brought to you for free by Swarthmore College Libraries' Works. It has been accepted for inclusion in Physics \& Astronomy Faculty Works by an authorized administrator of Works. For more information, please contact myworks@swarthmore.edu. 


\title{
A SINGLE CIRCUMBINARY DISK IN THE HD 98800 QUADRUPLE SYSTEM
}

\author{
D. W. Koerner, ${ }^{1}$ E. L. N. Jensen, ${ }^{2}$ K. L. Cruz, ${ }^{1}$ T. B. Guild,${ }^{1}$ and K. Gultekin ${ }^{1}$ \\ Received 1999 September 9; accepted 2000 February 10; published 2000 March 14
}

\begin{abstract}
We present subarcsecond thermal infrared imaging of HD 98800, a young quadruple system composed of a pair of low-mass spectroscopic binaries separated by 0.8 (38 AU), each with a K-dwarf primary. Images at wavelengths ranging from 5 to $24.5 \mu \mathrm{m}$ show unequivocally that the optically fainter binary, HD $98800 \mathrm{~B}$, is the sole source of a comparatively large infrared excess on which a silicate emission feature is superposed. The excess is detected only at wavelengths of $7.9 \mu \mathrm{m}$ and longer, peaks at $25 \mu \mathrm{m}$, and has a best-fit blackbody temperature of $150 \mathrm{~K}$, indicating that most of the dust lies at distances greater than the orbital separation of the spectroscopic binary. We estimate the radial extent of the dust with a disk model that approximates radiation from the spectroscopic binary as a single source of equivalent luminosity. Given the data, the most likely values of disk properties in the ranges considered are $R_{\text {in }}=5.0 \pm 2.5 \mathrm{AU}, \Delta R=13 \pm 8 \mathrm{AU}, \lambda_{0}=2_{-1.5}^{+4} \mu \mathrm{m}, \gamma=0 \pm$ 2.5 , and $\sigma_{\text {total }}=16 \pm 3 \mathrm{AU}^{2}$, where $R_{\text {in }}$ is the inner radius, $\Delta R$ is the radial extent of the disk, $\lambda_{0}$ is the effective grain size, $\gamma$ is the radial power-law exponent of the optical depth $\tau$, and $\sigma_{\text {total }}$ is the total cross section of the grains. The range of implied disk masses is $0.001-0.1$ times that of the Moon. These results show that, for a wide range of possible disk properties, a circumbinary disk is far more likely than a narrow ring.
\end{abstract}

Subject headings: binaries: close — binaries: spectroscopic — circumstellar matter — planetary systems — stars: imaging — stars: individual (HD 98800)

\section{INTRODUCTION}

The evolution of circumstellar dust around young stars is traced by a time-dependent signature in excess infrared emission. The evidence lies primarily in the spectral distribution of radiation as it chronicles infall from a protostellar envelope, viscous accretion in a gas-rich circumstellar disk, and dispersal in a dusty "debris disk" that survives as a last vestige of planet formation (Adams, Lada, \& Shu 1987; Backman \& Paresce 1993). Imaging has dramatically confirmed this interpretation, providing support for a standard model of circumstellar evolution and elucidating the role of circumstellar disks throughout the process (Beckwith \& Sargent 1996; Holland et al. 1998; Koerner 1997; Koerner et al. 1998). The coexistence of disks with stellar companions is attested to by a comparison of highresolution binary surveys (Ghez, Neugebauer, \& Matthews 1993; Leinert et al. 1993) with the results of imaging and longwavelength flux measurements (Jensen, Koerner, \& Mathieu 1996a; Jensen, Mathieu, \& Fuller 1996b; Mathieu et al. 2000). Disks are found to be reduced in mass for binaries with separations in the 10-100 AU range, similar to the typical disk size. However, circumstellar disks in binaries wider than 100 AU (Beckwith et al. 1990; Osterloh \& Beckwith 1995; Jensen et al. 1996b) and circumbinary disks around spectroscopic binaries (Jensen \& Mathieu 1997) are not obviously different from disks around single stars with respect to either their global properties or their frequency of occurrence. These results argue strongly for the possibility of an abundant and diverse population of extrasolar planets.

Among pre-main-sequence spectroscopic binaries with separations of $1 \mathrm{AU}$ or less, there is growing evidence that circumbinary disks are common. Massive circumbinary disks have been found in a handful of cases, demonstrating unequivocally that the presence of a small-separation binary is

\footnotetext{
${ }^{1}$ University of Pennsylvania, David Rittenhouse Laboratory, 209 South 33d Street, Philadelphia, PA 19104-6396.

${ }^{2}$ Department of Physics and Astronomy, Swarthmore College, 500 College Avenue, Swarthmore, PA 19081.
}

not an impediment to the formation of a protoplanetary disk (Jensen \& Mathieu 1997). Examples include GW Ori (Mathieu, Adams, \& Latham 1991; Mathieu et al. 1995), UZ Tau E (Jensen et al. 1996a; Mathieu, Martín, \& Maguzzu 1996), and DQ Tau (Mathieu et al. 1997), all with projected orbital separations of order $1 \mathrm{AU}$ or less and disk masses that are comparable to or greater than that estimated for the minimum-mass solar nebula.

The degree of complexity that is possible for multiple stardisk systems is perhaps nowhere better illustrated than in the case of the post-T Tauri quadruple system HD 98800. It is composed of a pair of low-mass spectroscopic binaries, each with a K-dwarf primary, that have a projected separation of 0"8 (37.6 AU at the 47 pc distance determined by Hipparcos) and estimated ages of $\sim 10 \mathrm{Myr}$ (Soderblom et al. 1998). Despite the presence of many stellar components, HD 98800 is associated with an unusually strong IRAS signature of dust emission with a temperature similar to the solar zodiacal dust bands (Walker \& Wolstencroft 1988; Zuckerman \& Becklin 1993; Sylvester et al. 1996) and with evidence for silicate emission from dust grains (Skinner, Barlow, \& Justtanont 1992). Until recently, there were no observations that provided a hint as to how this dust was distributed among the stellar components of the system. $N$-band imaging that marginally resolved the binary has now shown that most of the dust is associated with the optical secondary and spectroscopic binary HD 98800B (Gehrz et al. 1999). Here we present subarcsecond images from 5 to $25 \mu \mathrm{m}$ that fully resolve the 0 ".8 binary components of the HD 98800 system.

\section{OBSERVATIONS AND RESULTS}

HD 98800 was observed with JPL's mid-infrared camera MIRLIN at the f/40 bent-Cassegrain focus of the Keck II telescope on UT 1998 March 14. MIRLIN employs a Boeing $128 \times 128$ pixel, high-flux Si:As BIB detector with a plate scale at Keck II of 0.137 pixel $^{-1}$ and 17 ".5 field of view. Background subtraction was carried out by chopping the secondary mirror at an $\sim 4 \mathrm{~Hz}$ rate with $8^{\prime \prime}$ throw in the north-south di- 
TABLE 1

Component Flux Densities for the HD 98800 System

\begin{tabular}{cclcrrr}
\hline \hline $\begin{array}{c}\lambda_{\text {eff }} \\
\mu \mathrm{m})\end{array}$ & $\begin{array}{c}\delta \lambda \\
(\mu \mathrm{m})\end{array}$ & Calibrator/Flux $(\mathrm{Jy})$ & $\begin{array}{c}F_{\nu}(\mathrm{HD} 98800) \\
(\mathrm{Jy})\end{array}$ & $\begin{array}{c}\text { Component Ratio } \\
(\mathrm{B} / \mathrm{A})\end{array}$ & $\begin{array}{c}F_{\nu}(\mathrm{A}) \\
(\mathrm{Jy})\end{array}$ & $\begin{array}{c}F_{\nu}(\mathrm{B}) \\
(\mathrm{Jy})\end{array}$ \\
\hline $4.68 \ldots \ldots$ & 0.57 & $\beta \mathrm{Leo} / 28.95$ & $1.03 \pm 0.090^{\mathrm{a}}$ & 1.21 & $0.47 \pm 0.04$ & $0.72 \pm 0.04$ \\
$7.91 \ldots \ldots$. & 0.59 & $\beta \mathrm{Leo} / 10.68$ & $0.62 \pm 0.03$ & 2.46 & $0.18 \pm 0.02$ & $0.44 \pm 0.02$ \\
$8.81 \ldots \ldots$ & 0.87 & $\beta \mathrm{Leo} / 8.67$ & $0.10 \pm 0.04$ & 4.88 & $0.17 \pm 0.01$ & $0.83 \pm 0.03$ \\
$9.69 \ldots \ldots$. & 0.93 & $\beta \mathrm{Leo} / 7.21$ & $1.78 \pm 0.07$ & 10.20 & $0.15 \pm 0.02$ & $1.62 \pm 0.07$ \\
$10.27 \ldots \ldots$ & 1.01 & $\beta \mathrm{Leo} / 6.44$ & $2.02 \pm 0.08$ & 15.38 & $0.12 \pm 0.01$ & $1.90 \pm 0.08$ \\
$11.70 \ldots \ldots$ & 1.11 & $\beta \mathrm{Leo} / 5.00$ & $2.28 \pm 0.09$ & 21.28 & $0.10 \pm 0.01$ & $2.18 \pm 0.09$ \\
$12.49 \ldots \ldots$ & 1.16 & $\beta \mathrm{Leo} / 4.40$ & $2.19 \pm 0.09$ & 26.32 & $0.08 \pm 0.01$ & $2.02 \pm 0.08$ \\
$17.93 \ldots \ldots$ & 2.00 & $\alpha \mathrm{Hya} / 42.83$ & $4.98 \pm 0.20$ & $>27.78$ & $<0.174$ & $4.98 \pm 0.27$ \\
$20.81 \ldots \ldots$ & 1.65 & $\alpha \mathrm{Hya} / 31.74$ & $5.53 \pm 0.23$ & $>26.32$ & $<0.210$ & $5.53 \pm 0.31$ \\
$24.48 \ldots \ldots$ & 0.76 & $\alpha \mathrm{Hya} / 30.15$ & $8.62 \pm 0.35$ & $>47.62$ & $<0.181$ & $8.62 \pm 0.39$ \\
\hline
\end{tabular}

${ }^{\text {a }}$ Total flux taken from Zuckerman \& Becklin 1993.

rection and by nodding the telescope a similar distance eastwest after co-adding a few hundred chop pairs. Images of the source on the double-differenced frames were shifted and added to make the final $32 \times 32(4.4 \times 4 " 4)$ images. Observations were carried out at wavelengths from 4.7 to $24.5 \mu \mathrm{m}$ in the spectral bands listed in Table 1. Small dither steps were taken between chop-nod cycles. Infrared standards $\beta$ Leo (A3 V) and $\alpha$ Hya (K3 III) were observed in the same way at similar air masses.

The resulting images of HD 98800 are displayed in Figure 1. Since the half-maximum width of the point-spread function (PSF) is between 0".3 and 0".55 over the full wavelength range, it is possible to identify unambiguously the relative flux densities of the mid-infrared emission for the first time. Two point sources, separated by $0.81 \pm 0.02$, are detected at wavelengths up to $\lambda=12.5 \mu \mathrm{m}$ with an orientation that corresponds to the binary optical components, with A to the south and B to the north (Soderblom et al. 1998). Only a single point source is detected at the longest wavelengths. It is immediately apparent from the images that this emission arises predominantly from the northern source, corresponding to the optical secondary HD 98800B. In contrast, emission from the optical primary decreases steadily toward longer wavelengths. Separatecomponent flux densities were derived by fitting a measured PSF to each component and using the resulting flux component ratio to decompose the total flux into values for HD 98800A and B. The results are listed in Table 1 and plotted as a spectral energy distribution in Figure 2 together with measurements from the Hubble Space Telescope (HST), IRAS, and the James

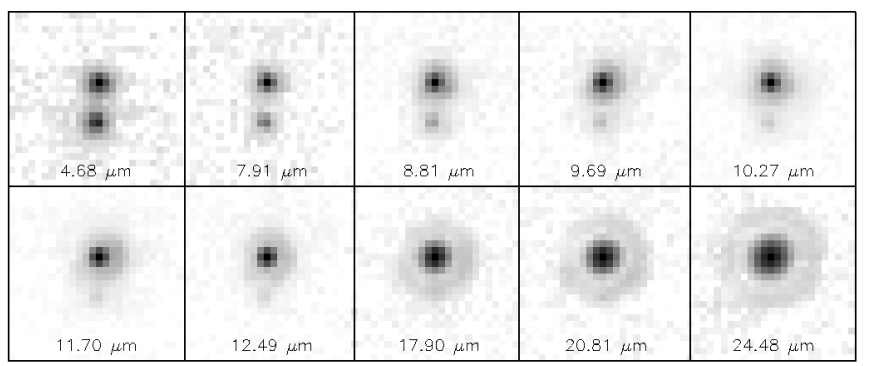

FIG. 1.-Keck/MIRLIN imaging of the thermal infrared emission from the HD 98800 quadruple system oriented with up axis aligned due north. The spectroscopic binaries HD 98800A and HD 98800B are clearly resolved from each other and are identified, respectively, with northern and southern point sources separated by 0.8 ( $38 \mathrm{AU}$ ). Emission from HD 98800A steadily decreases with wavelength as $\lambda^{-2}$ and is no longer detected in the $20 \mu \mathrm{m}$ images. In contrast, radiation from the optical secondary, HD 98800B, increases dramatically out to $24.5 \mu \mathrm{m}$.
Clerk Maxwell Telescope (JCMT) (Sylvester et al. 1996; Soderblom et al. 1998; Low, Hines, \& Schneider 1999). Midinfrared flux densities measured for the total system are in excellent agreement with earlier values published in the literature (Zuckerman \& Becklin 1993; Sylvester et al. 1996).

The distribution of mid-infrared flux between the components HD 98800A and B clearly indicates that the total infrared excess of the system is dominated by the contribution from HD 98800B. Values for the flux density of HD 98800B at $\lambda=12.5$ and $24.5 \mu \mathrm{m}$ agree very well with 12 and $25 \mu \mathrm{m}$ IRAS fluxes measured for the whole system. In contrast, flux densities for HD 98800A decrease approximately as $\lambda^{-2}$ between $\lambda=$ 7.9 and $12.5 \mu \mathrm{m}$, consistent with an origin in a stellar photosphere. At $12.5 \mu \mathrm{m}$, emission from HD 98800A contributes less than $4 \%$ of the total emission. At $24.5 \mu \mathrm{m}$, an upper limit to its contribution comprises only $2 \%$, and an estimated photospheric contribution only $0.2 \%$ of the total flux. It is thus a good approximation to ascribe all the emission from unresolved measurements at $\lambda>25 \mu \mathrm{m}$ to HD 98800B and neglect any contribution from HD 98800A. This result is largely in agreement with the conclusion of Gehrz et al. (1999), who nevertheless attributed some of the mid-infrared excess emission to HD 98800A on the basis of lower resolution imaging ( 1 " at $\lambda=9.8 \mu \mathrm{m}$ ) that only marginally resolved the 0 ".8 separation of components $\mathrm{A}$ and $\mathrm{B}$.

A spectral signature of silicate emission at $\lambda \approx 10 \mu \mathrm{m}$ is evident in the flux measurements of HD $98800 \mathrm{~B}$ plotted in Figure 2. It is displayed in more detail in Figure 3, where the measurements at 7.9 and $12.5 \mu \mathrm{m}$ have been assumed to represent featureless thermal continuum emission and a simple linear extrapolation between the two points has been subtracted off. The spectrum was then scaled to give the 7.9 and 12.5 um points a value of one to facilitate comparison with other data from the literature (see Hanner, Lynch, \& Russell 1994 for comparison of different continuum removal techniques). Silicate features from comets and interstellar dust are plotted in Figure 3 for comparison. It is readily apparent that the circumstellar dust feature resembles that from comets more than that from the interstellar medium. The feature is broader and does not show a single narrow peak between 9 and $10 \mu \mathrm{m}$ as seen for interstellar grains in the Trapezium. For comets, this broadened line shape has been interpreted as diagnostic of a mixture of amorphous and crystalline silicates that radiate predominantly at 9.8 and $11.2 \mu \mathrm{m}$, respectively (Hanner et al. 1994).

\section{MODELING AND DISCUSSION}

To better interpret the emission from HD 98800, we fitted model emission from stellar photospheres to optical (Wide Field 

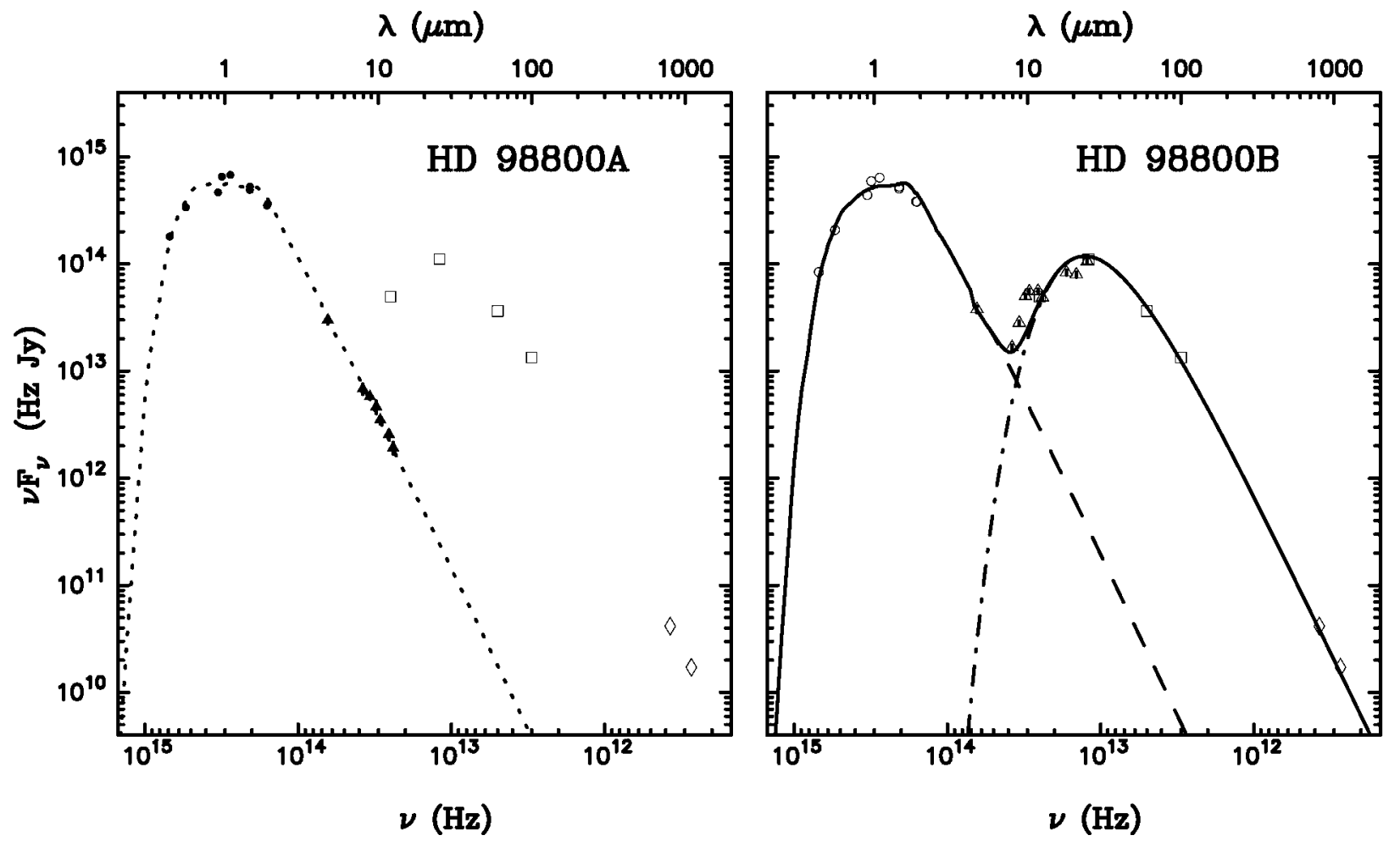

FIG. 2.- Spectral energy distributions for the separate components of HD 98800. The filled circles in the left panel represent HST (WFPC2 and NICMOS) fluxes for HD 98800A; the filled triangles represent the fluxes listed in Table 1. The dotted line is a model photosphere from a Kurucz fit to the HST data only. It clearly matches the mid-infrared fluxes presented in this work. The open squares and diamonds are plotted in both panels and represent IRAS and JCMT submillmeter fluxes, respectively, for the whole system. The open circles in the right panel represent $H S T$ fluxes for HD 98800B; the open triangles represent the fluxes listed in Table 1. The dashed line is a model photosphere fitted only to the HST fluxes for HD 98800A. The dot-dashed line represents emission from a model disk with parameters outlined in the text. Together with the photospheric model, it was fitted to measurements at 7.9 and $12.5 \mu \mathrm{m}$ and all longer wavelengths. The combined photospheric and disk emission is plotted as a solid line.

Planetary Camera 2 [WFPC2]) and near-infrared (Near-Infrared Camera Multiobject Spectrograph [NICMOS]) HST imaging that resolved components A and B (Soderblom et al. 1998; Low et al. 1999). These were matched by reddened model atmospheres from R. L. Kurucz by varying only the stellar luminosity, as described by Jensen \& Mathieu (1997). Multiple NICMOS measurements at roughly the same wavelength were averaged and weighted as a single point in the fit. Stellar effective temperatures were adopted from case $\mathrm{C}$ of Soderblom et al. (1998), where the single value $T_{\text {eff }}=4350 \mathrm{~K}$ was given for the spectroscopic binary HD $98800 \mathrm{~A}$, and $T_{\text {eff }}=4250$ and $3700 \mathrm{~K}$ were reported for the two stars in the double-lined spectroscopic binary HD 98800B. Soderblom et al. (1998) reported $A_{V}=0.44 \mathrm{mag}$ for $\mathrm{HD} 98800 \mathrm{~B}$ but gave no $A_{V}$ value for HD 98800A. We assumed $A_{V}=0$ for HD 98800A and used a standard interstellar extinction law with $A_{V}=3.1 E_{(B-V)}$ to redden the model for HD 98800B. The luminosity ratio of the two components was fixed at 2.7 based on the absolute $V$ magnitudes given by Soderblom et al. (1998) and the bolometric corrections from Kenyon \& Hartmann (1995). The best-fit models gave $L_{\text {star }}=0.78$ and $0.56 L_{\odot}$ and are plotted as a dotted and dashed line in Figure 2 for the A and B components, respectively.

An average dust temperature of $150 \mathrm{~K}$ was derived by fitting a Planck function to the excess continuum emission from HD 98800B, omitting points associated with the silicate feature. For grains $1-10 \mu \mathrm{m}$ in size, this temperature corresponds to a 4-12 AU distance from a single star of luminosity $0.56 L_{\odot}$. Given the $1 \mathrm{AU}$ orbital separation estimated for the components of HD 98800B (Soderblom et al. 1998), this temperature also implies that most of the dust is located in a circumbinary configuration around the spectroscopic binary. To estimate the radial extent of the dust, we also fitted the spectral energy distribution with a model of a disk around a single star of luminosity $0.56 L_{\odot}$. The model parameterization and fitting method are described in Koerner et al. (1998). Five parameters were varied in the fit, including inner radius $R_{\text {in }}$, radial extent $\Delta R$, effective particle size $\lambda_{0}$, and the radial power-law index $\gamma$ of the optical depth, $\tau(r)=\tau_{0}\left(r / r_{0}\right)^{-\gamma}$. The optical depth scaling $\tau_{0}$ was derived after varying the area-integrated optical depth, $\sigma_{\text {total }}=\int_{R_{\text {in }}}^{R_{\text {in }}+\Delta R} \tau_{0}\left(r / r_{0}\right)^{-\gamma} 2 \pi r d r$, an indicator of the total cross-sectional area of the grains. Parameter ranges considered were $0-9 \mathrm{AU}$ for $R_{\mathrm{in}}, 1-25 \mathrm{AU}$ for $\Delta R, 10^{-1}$ to $10^{3} \mu \mathrm{m}$ for $\lambda_{0},-4.0-4.0$ for $\gamma$, and 5-50 $\mathrm{AU}^{2}$ for $\sigma_{\text {total }}$. A disk model with the most likely values of these parameters is displayed in Figure 2; these are $R_{\text {in }}=5.0 \pm 2.5 \mathrm{AU}, \Delta R=13 \pm 8 \mathrm{AU}, \lambda_{0}=$ $2_{-1.5}^{+4} \mu \mathrm{m}, \gamma=0 \pm 2.5$, and $\sigma_{\text {total }}=16 \pm 3 \mathrm{AU}^{2}$, where the values quoted are central within a range of probabilities enclosing the $68 \%$ confidence level. The probability distribution is fairly flat within these ranges, and peak values are not always central but lie at $R_{\text {in }}=3.0 \mathrm{AU}, \Delta R=22 \mathrm{AU}, \lambda_{0}=1.8 \mu \mathrm{m}, \gamma=1$, and $\sigma_{\text {total }}=16 \mathrm{AU}^{2}$.

Many of these values are not narrowly constrained by the flux measurements alone, largely because the temperature dependence on both particle size and radial distance from the star makes it impossible to determine them uniquely. However, taken over the whole range of parameter space, there is greater than a $90 \%$ probability that the dust is distributed in a circum- 


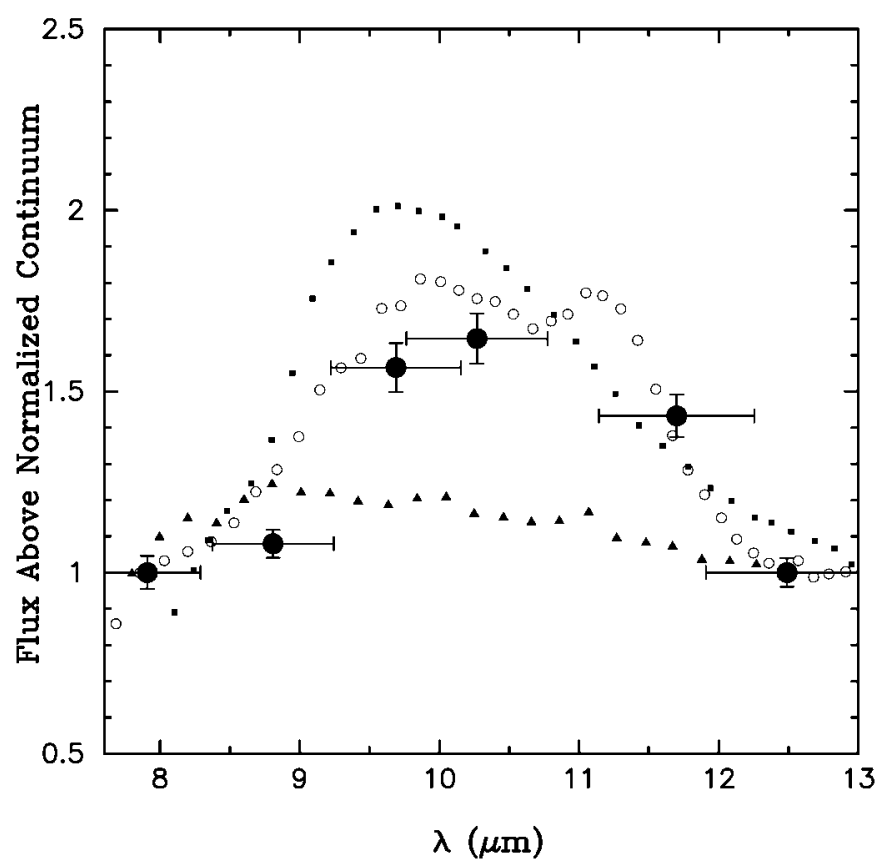

Fig. 3.-Plot of the emission from HD 98800B in the $10 \mu \mathrm{m}$ silicate band. The filled circles are derived from the flux densities that are listed in Table 1 by the subtraction of continuum emission interpolated between the points at $\lambda=7.9$ and $12.5 \mu \mathrm{m}$. The vertical error bars are derived from uncertainties listed in Table 1. The horizontal error bars refer only to the filter widths, also given in Table 1. The filled squares depict the silicate emission feature as observed in the interstellar medium toward the Trapezium. The filled triangles and open circles refer to measurements taken from Hanner et al. (1994) for comets Levy and Austin, respectively.

binary disk, with $\Delta R / R_{\text {in }}>1$, rather than a narrow ring like that around HR 4796A $\left(\Delta R / R_{\text {in }}<0.25\right.$; Koerner et al. 1998; Schneider et al. 1999). We emphasize the caveat that these estimates apply only under the assumptions of this particular disk model. An estimate of the true inner radius, for example, should take into account radiation from the two stellar components, and some temperature broadening may be due to a range of emissivities inherent in an unknown particle-size distribution. However, these effects are unlikely to alter our gen- eral conclusion about the disk versus ringlike nature of the dust.

The total cross section for dust grains around HD 98800B, $\sigma_{\text {total }}=16 \pm 3 \mathrm{AU}^{2}$, is $2-3$ orders of magnitude smaller than for several other debris disks (e.g., $\beta$ Pic, HR 4796A, and 49 Cet). Thus, from the standpoint of circumstellar mass, the disk around HD 98800B is not as remarkable as suggested by the infrared excess alone (Zuckerman \& Becklin 1993). The relatively high fractional luminosity is, instead, a consequence of the dust location close to the star where grains intercept a greater fraction of the stellar radiation. Assuming a range of plausible grain densities, $\rho=1.0-3.0 \mathrm{~g} \mathrm{~cm}^{-3}$, values of $\sigma_{\text {total }}$ and $\lambda_{0}$ (grain radius $a=\lambda_{0} / 1.5$; cf. Backman, Gillett, \& Witteborn 1992) imply a disk mass in the range of 0.001-0.1 lunar masses.

Models that incorporate a circumbinary disk surrounding an optically thin region of warmer dust have served to explain the spectral energy distributions of younger T Tauri spectroscopic binaries (Jensen \& Mathieu 1997). It is likely that HD 98800B is a similar system in a later phase of evolution. Modeling of the circumbinary dust emission indicates the location of the dust in a radial zone associated with planet building early in the life of our own solar system. Consequently, it may well represent the telltale signature of planet formation in a hierarchically ordered multiple star system. If so, we can expect our picture of the plenitude and diversity of extrasolar planetary systems to become increasingly rich as it is revealed by impending surveys with high-resolution techniques now under development.

We gratefully acknowledge the support of the NSF's "Life in Extreme Environments" program through grant AST 9714246. Data presented herein were obtained at the W. M. Keck Observatory (WMKO), which is operated as a scientific partnership among the California Institute of Technology, the University of California, and the National Aeronautics and Space Administration. The Observatory was made possible by the generous financial support of the W. M. Keck Foundation. We wish to thank an anonymous referee for useful comments. A great debt is due, also, to Robert Goodrich and the WMKO summit staff for their many hours of assistance in adapting MIRLIN to the Keck II visitor instrument port.

\section{REFERENCES}

Adams, F. C., Lada, C. J., \& Shu, F. H. 1987, ApJ, 312, 788

Backman, D. E., Gillett, F. C., \& Witteborn, F. C. 1992, ApJ, 385, 670

Backman, D. E., \& Paresce, F. 1993, in Protostars and Planets III, ed. E. H.

Levy \& J. I. Lunine (Tucson: Univ. Arizona Press), 1253

Beckwith, S. V. W., \& Sargent, A. I. 1996, Nature, 383, 189

Beckwith, S. V. W., Sargent, A. I., Chini, R. S., \& Güsten, R. 1990, AJ, 99, 924

Gehrz, R. D., Smith, N., Low, F. J., Krautter, J., Nollenberg, J. G., \& Jones, T. J. 1999, ApJ, 512, L55

Ghez, A. M., Neugebauer, G., \& Matthews, K. 1993, AJ, 106, 2005

Hanner, M. S., Lynch, D. K., \& Russell, R. W. 1994, ApJ, 425, 274

Holland, W. S., et al. 1998, Nature, 382, 788

Jensen, E. L. N., Koerner, D. W., \& Mathieu, R. D. 1996a, AJ, 111, 2431

Jensen, E. L. N., \& Mathieu, R. D. 1997, AJ, 114, 301

Jensen, E. L. N., Mathieu, R. D., \& Fuller, G. A. 1996b, ApJ, 458, 312

Kenyon, S. J., \& Hartmann, L. 1995, ApJS, 101, 117

Koerner, D.W. 1997, Origins Life Evol. Biosphere, 27, 157

Koerner, D. W., Ressler, M. E., Werner, M. W., \& Backman, D. E. 1998, ApJ, 503, L83
Leinert, Ch., Zinnecker, H., Weitzel, N., Christou, J., Ridgway, S. T., Jameson, R., Haas, M., \& Lenzen, R. 1993, A\&A, 278, 129

Low, F. J., Hines, D. C., \& Schneider, G. 1999, ApJ, 520, L45

Mathieu, R. D., Adams, F. C., Fuller, G. A., Jensen, E. L. N., Koerner, D. W., \& Sargent, A. I. 1995, AJ, 109, 2655

Mathieu, R. D., Adams, F. C., \& Latham, D. W. 1991, AJ, 101, 2184

Mathieu, R. D., Ghez, A. M., Jensen, E. L. N., \& Simon, M. 2000, in Protostars and Planets IV, ed. V. Mannings, A. Boss, \& S. Russell (Tucson: Univ. Arizona Press), in press

Mathieu, R. D., Martín, E. L., \& Maguzzu, A. 1996, BAAS, 28, 920

Mathieu, R. D., Stassun, K., Basri, G., Jensen, E. L. N., Johns-Krull, C. M., Valenti, J. A., \& Hartmann, L. W. 1997, AJ, 113, 1841

Osterloh, M., \& Beckwith, S. V. W. 1995, ApJ, 439, 288

Schneider, G., et al. 1999, ApJ, 513, L127

Skinner, S. L., Barlow, M. J., \& Justtanont, K. 1992, MNRAS, 255, 31

Soderblom, D. R., et al. 1998, ApJ, 498, 385

Sylvester, R. J., Skinner, C. J., Barlow, M. J., \& Mannings, V. 1996, MNRAS, 279, 915

Walker, H. J., \& Wolstencroft, R. D. 1988, PASP, 100, 1509

Zuckerman, B., \& Becklin, E. E. 1993, ApJ, 406, L25 